\title{
LRRC15 is differentially expressed in brain metastatic breast cancer.
}

Shahan Mamoor, MS 1

1shahanmamoor@gmail.com

East Islip, NY USA

One study has reported a $34 \%$ incidence of central nervous system metastases, including metastasis to the brain, in breast cancer patients treated with trastuzumab ${ }^{1}$. We mined published microarray data ${ }^{2,3}$ to discover genes associated with brain metastasis in breast cancer. We identified significant differential expression of the leucine-rich repeat-containing protein 15, encoded by LRRC154,5, in the brain metastases of patients with metastatic breast cancer. LRRC15 may be relevant to the biology underlying colonization of the brain with metastatic breast cancer clones.

Keyword: breast cancer, metastasis, brain metastasis, central nervous system metastasis, LRRC15, leucine-rich repeat-containing protein 15 , systems biology of breast cancer, targeted therapeutics in breast cancer. 
One study reported a 34\% incidence of central nervous system metastases in patients with breast cancer treated with the monoclonal antibody trastuzumab (Herceptin) ${ }^{1}$. Treatment options for patients with brain metastatic breast cancer are limited ${ }^{6}$. We mined published microarray datasets ${ }^{2,3}$ to understand in an unbiased and systems-level fashion genes whose expression most significantly distinguished brain metastases from primary tumors of the breast, the cells from which these metastatic clones are presumably derived. We found significant differential expression of LRRC15 in the brain metastases of patients with brain metastatic breast cancer.

\section{Methods}

We utilized microarray datasets GSE $125989^{2}$ and GSE100534³ for this differential gene expression analysis of brain metastatic breast cancer in conjunction with GEO2R. GSE100534 was generated using Affymetrix Human Gene 1.0 ST Array technology with $n=16$ primary tumors from patients with breast cancer and $n=3$ brain metastases from patients with brain metastatic breast cancer. GSE43837 was generated using Affymetrix Human X3P Array technology with $n=19$ primary breast tumors and $n=19$ brain metastases from patients with breast cancer, analysis performed using platform GPL1352. The Benjamini and Hochberg method of $p$-value adjustment was used for ranking of differential expression but raw $p$-values were used to assess statistical significance of global differential expression. Log-transformation of data was auto-detected, and the NCBI generated category of platform annotation was used. A statistical test was performed to evaluate whether LRRC15 gene expression was significantly between primary breast tumors and brain metastases in humans with breast cancer using a two-tailed, unpaired t-test with Welch's correction. We used PRISM for all statistical analyses of differential gene expression in human breast cancer (Version 8.4.0)(455).

\section{Results}

We mined published microarray data ${ }^{2,3}$ to discover genes associated with brain metastasis in breast cancer.

\section{LRRC15 is differentially expressed in brain metastatic breast cancer.}

We identified the leucine-rich repeat-containing protein 15, encoded by LRRC15, as among the genes whose expression changes most significantly when comparing brain metastases to primary tumors of the breast in patients with brain metastatic breast cancer ${ }^{2}$ (Table 1). When sorting each of the genes expressed in brain metastases based on significance of change as compared to primary tumors of the breast, LRRC15 ranked 73 out of 22277 total transcripts (Table 1), equating to $99.7 \%$ differential expression. Differential expression of LRRC15 in brain metastases in brain metastatic breast cancer was statistically significant (Table $1 ; p=1.72 \mathrm{E}-04)$.

We queried a second microarray dataset ${ }^{3}$ to validate transcriptome-wide differential expression of LRRC15 in brain metastatic breast cancer. We again found that LRRC15 was among the genes whose expression changed most significantly when comparing metastases of the brain to primary tumors of the breast in patients with breast cancer (Table 2). When sorting each of the genes expressed in brain metastases based on significance of change as compared to primary tumors of the breast, LRRC15 ranked 4863 out of 33297 total transcripts (Table 2), equating to $85.4 \%$ differential expression. Differential expression of LRRC15 in brain metastases in brain metastatic breast cancer was statistically significant (Table $2 ; p=1.52 \mathrm{E}-02)$. 
LRRC15 is expressed at significantly lower levels in brain metastases as compared to primary tumors of the breast in brain metastatic breast cancer.

We obtained exact mRNA expression values for LRRC15 from brain metastases and primary tumors of the breast to evaluate magnitude and direction of change in LRRC15 expression in brain metastases in patients with breast cancer. LRRC15 was expressed at lower levels in brain metastases in patients with breast cancer and this difference was statistically significant (Figure $1 ; p=0.0007$ ). We calculated a mean fold change of $0.76 \pm 0.19$ in LRRC15 expression when comparing brain metastatic tissues to primary tumors of the breast (Table 1).

Thus, we found that LRRC15 was among the genes, globally, whose expression was most different when comparing primary tumors of the breast to brain metastases in patients with breast cancer, and present at significantly lower quantities at the mRNA level in brain metastatic tissues as compared to primary tumors of the breast.

\section{Discussion}

We report here that the gene encoding the leucine-rich repeat-containing protein 15, LRRC15, was among those whose expression was most quantitatively different in metastases to the brain when compared to primary tumors of the breast in patients with metastatic breast cancer. An antibody targeting LRRC15 has demonstrated some level of efficacy in a phase I clinical trial in solid tumors including sarcomas5. LRRC15 may be of relevance to the biology underlying any number of steps important for metastasis to the brain: exit from the breast, traversing the vasculature and the blood-brain barrier, and colonization of the brain. 


\section{References}

1. Bendell, J.C., Domchek, S.M., Burstein, H.J., Harris, L., Younger, J., Kuter, I., Bunnell, C., Rue, M., Gelman, R. and Winer, E., 2003. Central nervous system metastases in women who receive trastuzumab-based therapy for metastatic breast carcinoma. Cancer, 97(12), pp.2972-2977.

2. Iwamoto, T., Niikura, N., Ogiya, R., Yasojima, H., Watanabe, K.I., Kanbayashi, C., Tsuneizumi, M., Matsui, A., Fujisawa, T., Iwasa, T. and Shien, T., 2019. Distinct gene expression profiles between primary breast cancers and brain metastases from pair-matched samples. Scientific reports, 9(1), pp. 1-8.

3. Schulten, H.J., Bangash, M., Karim, S., Dallol, A., Hussein, D., Merdad, A., Al-Thoubaity, F.K., AlMaghrabi, J., Jamal, A., Al-Ghamdi, F. and Choudhry, H., 2017. Comprehensive molecular biomarker identification in breast cancer brain metastases. Journal of translational medicine, 15(1), p.269.

4. Schuetz, C., Fehm, T., Solomayer, E., Wallwiener, D. and Neubauer, H., 2007. LRRC15 in breast cancer: Description of two transcript isoforms and identification of genotypical polymorphisms in cells lines with different invasion abilities.

5. Demetri, G.D., Luke, J.J., Hollebecque, A., Powderly, J.D., Spira, A.I., Subbiah, V., Lai, D.W., Yue, H., Kasichayanula, S., Gulbranson, S. and Purcell, J., 2019. First-in-human phase 1 study of ABBV-085, an antibody-drug conjugate (ADC) targeting LRRC15, in sarcomas and other advanced solid tumors. 
PAGE 5 
1

2

\begin{tabular}{c|l|l|l|l|l|l|}
\hline Rank & ID & p-value & t & B & Gene & Gene name \\
\hline $\mathbf{4 8 6 3}$ & 8092839 & $1.52 \mathrm{E}-02$ & -2.6526676 & -3.393735 & LRRC15 & $\begin{array}{l}\text { leucine rich repeat } \\
\text { containing 15 }\end{array}$ \\
\hline
\end{tabular}

Table 2: LRRC15 is differentially expressed in brain metastatic breast cancer.

Rank of differential expression, probe ID, $p$-value with respect to differential expression, $t$, a moderated tstatistic, B, the log-odds of differential expression between the two groups compared, gene and gene name are listed in this chart.

PAGE 6 


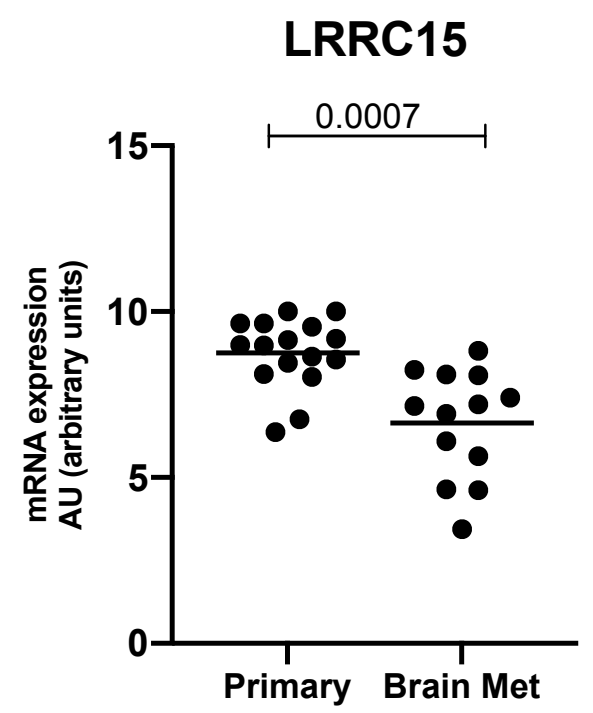

Figure 1: LRRC15 is expressed at significantly lower levels in brain metastases as compared to primary tumors of the breast in patients with brain metastatic breast cancer.

The mRNA expression level of LRRC15 in primary tumors of the breast (left) and in the brain metastases of brain metastatic breast cancer patients (right) is graphically represented here with mean LRRC15 levels marked and the result of a statistical test evaluating difference in mRNA expression level between brain metastases and primary tumors of the breast, a $p$-value, listed above. 